\title{
Bribery and public procurement: an experimental study
}

\author{
Susanne Büchner • Andreas Freytag • \\ Luis G. González • Werner Güth
}

Received: 18 September 2006 / Accepted: 20 February 2008 / Published online: 8 May 2008

(C) The Author(s) 2008. This article is published with open access at Springerlink.com

\begin{abstract}
A procurement contract is granted by a bureaucrat (the auctioneer) who is interested in a low price and a bribe from the provider. Procurement is thus a multi-dimensional bidding contest with one-dimensional type space (the privately known cost). The optimal price and bribe bid is derived based on an iid private cost assumption. In the experiment, bribes are negatively framed to capture that society is better off if bribes are rare or low. Although bid prices are lower than predicted, behavior is qualitatively in line with the linear equilibrium prediction. When bribes generate a negative externality, there is a significant increase in the variability of the data.
\end{abstract}

Keywords Corruption · Procurement auctions

\section{Introduction}

Bureaucrats with discretionary power to select suppliers in procurement contests are arguably more prone to accept (or even require) bribes when granting a contract. Indeed, reports on bribe-taking are frequently at the center of efforts to reform the governance structures of public procurement (see, e.g., Karahan et al. 2002). However, in spite of the many theoretical and empirical analyses of corruption already available in the literature (for an

\footnotetext{
S. Büchner · W. Güth (凶)

Max Planck Institute of Economics, Kahlaische Strasse 10, 07745 Jena, Germany e-mail: gueth@econ.mpg.de

S. Büchner

e-mail: buechner@econ.mpg.de
}

\section{A. Freytag}

Friedrich Schiller University of Jena, Carl-Zeiss-Strasse 3, 07743 Jena, Germany

e-mail: a.freytag@wiwi.uni-jena.de

\section{L.G. González}

United Nations, New York, NY, USA

e-mail: gonzalezmorales@gmail.com 
overview see, e.g., Rose-Ackerman 1999; OECD 2007), bribing is a difficult phenomenon to prove and to study in detail, as its illegal nature provides strong incentives for concealment.

Looking at recent events-e.g., even in Japanese sumo wrestling (Duggan and Levitt 2002) and, more recently, in Italian football—one would have the impression that corruption permeates today's world. Questionnaires like those used in calculating the corruption perception index (Transparency International 2007) indicate that corruption is wide-spread, and not exclusively a developing world phenomenon. It also seems evident that corruption is extremely costly and an obstacle to economic efficiency and economic development, although its true costs are difficult to calculate and general assessments must remain rather speculative. Since corruption in public procurement is particularly difficult to observe empirically, it is reasonable to assume that the available evidence on bribes actually offered to bureaucrats is only the tip of an iceberg. Given its hidden nature, therefore, experiments are an alternative to study corruption in public procurement, as one can simulate a procurement auction under more or less realistic circumstances.

One way to model corruption in procurement auctions is to focus on the ex post collusion opportunities between a corrupt bureaucrat and one specific bidder, who is given the opportunity to re-submit his or her bid after the bureaucrat has received all (initial) bids. This re-negotiation approach is followed, for instance, by Compte et al. (2005) and by Lengwiler and Wolfstetter (2006). In contrast, in our theoretical and experimental analysis we assume that posting lower prices and offering larger bribes are the two possible ways in which potential suppliers can increase their own chances of obtaining the contract. In doing so, we refrain from specifying how they negotiate with the bureaucrat over whether and what kind of bribes to pay. Instead, we focus on the way in which different bidders solve the tradeoff between price-oriented and bribe-oriented competition, and then proceed to investigate how they react to the presence (or absence) of negative externalities affecting all members of society when bribing takes place. Thus we address the empirical question whether ethical considerations have any effect on bribing behavior.

Our model of procurement and corruption is similar to those of Beck and Maher (1986) and of Lien (1986), who established the fundamental isomorphism between bribes and pricecompetition. However, our setup reflects a situation where the bidders compete by choosing prices and bribes simultaneously (as in Burguet and Che 2004, and Arozamena and Weinschelbaum 2004), whereby the auctioneer's commonly known preferences reflect institutionalized corruption. This renders the bidding contest a two-dimensional auction (Che 1993), where bidders' types are defined by their privately known, one-dimensional cost. In other words, by assuming exogenous preferences of the bureacrat, we study the behavior of the potential providers when facing a corrupt bureacracy, and not how bureacrats solve the conflict between their moral duties and their desire for bribes. Rather than trying to explore the model in full generality, e.g., for at least two bidders and all possible iid-priors, we focus on the experimentally implemented version with only two bidders and uniform piors. In fact, our bidding scenario could also be interpreted as one where sellers compete in price and quality, which is appropriate since a corrupt bureaucrat faces exactly such a tradeoff between "price" and "quality" when selecting a provider. Building on the theoretical framework of a two-dimensional action model, our laboratory experiment then captures the ethical aspect of corruption by attaching a negative externality to bribing as an experimental treatment.

Below we introduce the game model and derive its linear equilibrium (Sect. 2). We then describe the experimental procedure (Sect. 3) and analyze the experimental data (Sect. 4). Section 5 concludes. 


\section{The model}

Let two a priori symmetric bidders compete for a public procurement contract to provide an indivisible good. If the contract is granted to any of the two bidders, there would be an increase of $\gamma>0$ in social welfare with respect to some status-quo level. Thus, if the final price paid to the supplier of the good is $p$, the social value of granting the procurement contract is $V(p)=\gamma-p$ (without taking into account the winning bidder's profit).

The bureaucrat, being society's agent, is in charge of evaluating competing bids and choosing the provider. Although the bureaucrat's duty is to maximize $V(p)$, he also takes into consideration any bribes accompanying the price bids. In particular, denoting by $b$ a bribe offered along with a price bid $p$, the bureaucrat will select an offer $(p, b)$ over the alternative $\left(p^{\prime}, b^{\prime}\right)$ if

$$
U(p, b)>U\left(p^{\prime}, b^{\prime}\right),
$$

where $U(p, b)=(\theta+b) V(p)$ so that the parameter $\theta>0$, together with $p<\gamma$, guarantees monotonicity of $U(p, b)$ in its two arguments. Obviously the bureaucrat faces a tradeoff, since he is interested in both a low price and a high bribe, and the marginal utility of a price decrease negatively depends on the bribe offered (and vice-versa).

The choice of the utility function above has been made to preserve generality while being easy to implement (and understand) in an experimental setting. In Appendix, it is shown that nothing much changes if the behavior of the bureaucracy is in line with the more general criterion $(\theta+b)^{\beta} V(p)^{\alpha}>\left(\theta+b^{\prime}\right)^{\beta} V\left(p^{\prime}\right)^{\alpha}$, i.e., with more complex utility functions $U(p, b)=(\theta+b)^{\beta} V(p)^{\alpha}$ of which $\alpha=1=\beta$ is only a particular case. On the other hand, whereas choosing an additive and strictly convex utility specification is straightforward in theory, it would very likely have overburdened participants in the experiment.

The more complex utility function $U(p, b)$ above satisfies $\frac{\partial}{\partial b} U(p, b)>0$ as long as $\beta>0$. Thus it only allows one to approximate honesty by letting $\beta$ converge to 0 . Furthermore, for $V(p)=\gamma-p$, one has not only

$$
\frac{\partial}{\partial b} U(p, b)=\beta(\theta+b)^{\beta-1}(\gamma-p)^{\alpha}>0,
$$

but also

$$
\frac{\partial^{2}}{\partial b \partial p} U(p, b)=-\alpha \beta(\theta+b)^{\beta-1}(\gamma-p)^{\alpha-1}<0 .
$$

Thus, the bureaucrat always gains by a larger bribe, although the marginal effect decreases the higher the price, i.e., the more society is harmed.

As suggested by one of our anonymous referees, one could also use a more flexible bureaucrat's utility function of the additive form

$$
U(p, b)=\theta V(p)+f(V(p)) b
$$

with $\theta>0$ and $V(p)=\gamma-p$ as above. Total honesty would then be captured by $f(\cdot) \equiv 0$, whereas $f(\cdot) \equiv r>0$ would imply that the marginal utility of $b$ is independent of how much society suffers, i.e., of how large the price $p$ is. In our multiplicative formulation, the first case can only be approximated by $\beta \rightarrow 0$ and $\alpha=1$, while the second case would require $\alpha \beta \rightarrow 0$. In our view, the additive form of $U(p, b)$ might be especially appropriate for an experimental setting in which the bureaucrat's role is actually played by a participant (see Sect. 3 for details on our experimental design). This participant could, for instance, specify 
some affine function $f(V(p))$ by choosing a slope and a constant, which then would be revealed to the bidders. One would thus allow for the endogenous determination of $U(p, b)$ and still derive equilibrium benchmarks for the bidding contests. By allowing the bureaucrat participant to adjust his or her behavior less frequently than the bidders, it would be possible to distinguish pure learning of fixed bidding rules from adjustments made in the face of institutional changes. Furthermore, to capture the referee's intuition, the bureaucrat could choose a charity whose donations, to be paid by the experimenters, get smaller the larger the price.

The costs of both bidders, $C$ and $C^{\prime}$, are two iid random variables with Uniform[0,1] distribution. While the rules of the procurement auction necessarily remain silent about the feasible levels of the bribe component of a bid, $b$, they impose an upper bound $\bar{p}<\gamma$ on the price component. Therefore bidders can only submit price bids $p, p^{\prime} \in[0, \bar{p}]$. It is also assumed that $\theta>\bar{p}$, so that the bureaucrat always has a strict preference for lower prices other things being equal (thus never deviating radically from his moral duties).

We only consider the "price bid = price paid" rule, in which the winning bidder receives the price specified in his own offer. Thus, if a provider with actual cost $C$ submits an offer $(p, b)$ while his competitor's offer is equal to $\left(p^{\prime}, b^{\prime}\right)$, then the former earns

$$
\pi=p-C-b,
$$

provided that (1) holds. The losing competitor, who offered $\left(p^{\prime}, b^{\prime}\right)$, is left empty handed and earns zero (i.e., an offered bribe is paid only by the provider).

Due to the symmetry of both suppliers we focus on the symmetric equilibrium solution of the game that satisfies some obvious monotonicity properties. In particular, we restrict our analysis to linear solutions in the sense of linear functions $p(\cdot)$ and $b(\cdot)$ of the cost level $C$ for risk-neutral bidders.

Proposition 1 In the procurement contest with bribes there is a unique symmetric and linear equilibrium $\left(p^{*}(C), b^{*}(C)\right)$ such that $p^{*}(C)-b^{*}(C)$ increases with $C$, namely

$$
b^{*}(C)=\frac{\gamma-\theta}{2}-\frac{1+C}{4}
$$

and

$$
p^{*}(C)=\frac{\gamma-\theta}{2}+\frac{1+C}{4}
$$

Proof The problem of deciding how much to bid (the choice of $0 \leq p \leq \bar{p}$ ) and how much to offer as a bribe (the choice of $b \geq 0$ ) can be solved in two steps: a supplier can first identify the set of offers that maximize his acceptance probability by the bureaucracy, and then choose $p$ and $b$ from this set so as to maximize his expected profit. Let us start with the first problem: For given cost $C$, any winning offer $(p, b)$ with constant net revenue $p-b=k$ yields the same profit $\pi(C, k)=k-C$. Maximizing acceptance probability thus means solving

$$
\max _{p, b} U(p, b) \quad \text { s.t. } \quad p-b=k,
$$

which yields

$$
b^{*}(k)= \begin{cases}\frac{\gamma-\theta}{2}-\frac{k}{2}, & \text { if } k \in[0, \gamma-\theta], \\ 0, & \text { if } k \in(\gamma-\theta, \bar{p}]\end{cases}
$$


and

$$
p^{*}(k)= \begin{cases}\frac{\gamma-\theta}{2}+\frac{k}{2}, & \text { if } k \in[0, \gamma-\theta], \\ k, & \text { if } k \in(\gamma-\theta, \bar{p}] .\end{cases}
$$

Thus, what remains to be determined is the function $k=k(C)$ which allows writing $p^{*}$ and $b^{*}$ as functions $p^{*}(C)$ and $b^{*}(C)$. Notice that

$$
U\left(p^{*}(k(C)), b^{*}(k(C))\right)>U\left(p^{*}\left(k\left(C^{\prime}\right)\right), b^{*}\left(k\left(C^{\prime}\right)\right)\right)
$$

if and only if

$$
k\left(C^{\prime}\right)>k(C) .
$$

For a monotonic (and hence invertible) function $k(\cdot)$ with $k^{\prime}(\cdot)>0$, we can therefore express a bidder's payoff, based on $p^{*}$ and $b^{*}$, as

$$
\begin{aligned}
\pi(\hat{k}) & =\int_{C^{\prime}>k^{-1}(\hat{k})}(\hat{k}-C) d C^{\prime} \\
& =(\hat{k}-C)\left(1-k^{-1}(\hat{k})\right),
\end{aligned}
$$

where $\hat{k}=k(C)$. Then, from $\pi^{\prime}(\hat{k})=0$, one derives

$$
\frac{d}{d \hat{k}} k^{-1}(\hat{k})=\frac{1-k^{-1}(\hat{k})}{\hat{k}-C}=\frac{1-k^{-1}(\hat{k})}{\hat{k}-k^{-1}(\hat{k})}
$$

or for $f(\hat{k})=C(\hat{k})$,

$$
f^{\prime}(\hat{k})=\frac{1-f(\hat{k})}{\hat{k}-f(\hat{k})} .
$$

The linear solution of this differential equation is

$$
f(\hat{k})=2 \hat{k}-1 \text {. }
$$

Setting $C=f(\hat{k})$ yields

$$
k^{*}(C)=\frac{1+C}{2},
$$

justifying the initial assumption of monotonicity. Thus,

$$
b^{*}(C)=\frac{\gamma-\theta}{2}-\frac{1+C}{4} \quad \text { and } \quad p^{*}(C)=\frac{\gamma-\theta}{2}+\frac{1+C}{4}
$$

is the unique symmetric, linearly monotonic equilibrium.

Since $p^{*}(C)-b^{*}(C)=\frac{1+C}{2}$, the two preference parameters $\theta$ and $w_{0}$ of the bureaucrat (see Appendix) affect the equilibrium choices $b^{*}(C)$ and $p^{*}(C)$, but not their difference. One could explore this statistically by regressing $p^{*}(C)-b^{*}(C)$ linearly as a function of $C$ while varying the parameters $\gamma$ and $\theta$ (which we guarantee the monotonicity of $U(\cdot)$ in both its arguments). 


\section{Experimental design}

To compare the predicted price-bribe combinations against actual choice behavior, we implemented in a laboratory experiment the auction mechanism described above. In doing so, we only assumed an impersonal-and more or less corrupt-bureaucratic institution, enforcing decision rule (1) automatically and without any real participants playing the role of a bureaucrat. This allowed us to focus only on how potential providers react to a corrupt bureaucracy while avoiding loss of experimental control.

In the experiment, the random cost variable assigned to each participant in each period was iid Uniform[0,100], and the maximum price was set equal to $\bar{p}=150$ tokens. To prevent participants from making losses, they were constrained to submit bids $(p, b)$ such that $p-$ $C-b \geq 0$. The parameters of the allocation function $U(p, b)$ were set such that $\gamma=160$ tokens and $\theta=10$ tokens. Thus, the benchmark bid functions are

$$
b^{*}(C)=50-\frac{1}{4} C
$$

and

$$
p^{*}(C)=100+\frac{1}{4} C
$$

for all $C \in[0,100]$.

\subsection{Matching protocols}

A total of 114 participants were divided in four experimental sessions, whereby the first three sessions had 30 participants each, while session 4 consisted of 24 participants only. Participants played the auction game in pairs over 30 rounds, using either a partners' protocol (sessions 1 and 4) or (an anonymous) random matching protocol (sessions 2 and 3). In case of the partners' protocol, subjects played with the same partner ten periods, so that each participant played with three different partners during the whole session. In case of the random matching protocol, pairs of participants were re-matched each period within matching groups of size six.

By the strangers' protocol we explore the case where, as in the theoretical analysis, no repeated interaction takes place. The partners' protocol captures the idea that often the same suppliers compete in successive procurement auctions. Although the benchmark solution can be justified even for the partners' design with repeated interaction given that the finite horizon is commonly known, one might object that initial cooperation is a well-known phenomenon that contradicts backwards rationality (see Selten and Stoecker 1986, for an early confirmation). The effect of repeated interaction in this particular setting remains an open question, however, since reputation effects apply so far only to deterministic games. For games with private information (see the survey of Kagel 1995, on auction experiments) we are not aware of any reliable evidence of this behavioral regularity. If, contrary to our expectations, the strangers' protocol would trigger reputation effects, this should show reputation via restart effects, i.e., by a revival of cooperation when confronting a new partner.

\subsection{Fixed versus variable exchange rates}

We also used the exchange rate between tokens and Euros as a treatment variable in order to implement the negative external effects created by bribery. Whereas sessions 1 and 2 
relied on a fixed exchange rate of 100 tokens $=2.50$ Euros, sessions 3 and 4 implemented a variable exchange rate, which could take on values of either 2.00 or 3.00 Euros per 100 tokens. In the latter case, the payments of each matching group were calculated every ten rounds in the following way: The total amount of bribes offered by all members of each matching group during a block of ten rounds was compared with the respective total amount offered by a "reference" matching group. If the players in the matching group in question offered higher bribes than the players in its reference group, then the former were paid 2.00 Euros for each 100 tokens they had earned during the block of ten rounds. If they offered lower bribes than the reference group, then they received 3.00 Euros for each 100 tokens they earned during the block of ten rounds. The reference matching group used to do this comparison was randomly chosen (for each matching group) every ten periods among the remaining four groups in the session.

The variable exchange rates protocol is intended to capture institutional competition in the sense that countries, regions, or industries facing less corrupt bureaucracies will, for instance, be more attractive for investors in the long run, while those with corrupt institutions will suffer a comparative disadvantage. One may ask whether or not this institutional competition raises questions about our benchmark solution. However, provided that cardinal utilities are linear in monetary earnings, a change in the value of one token is only a positively affine transformation of utility. Thus the major concern is the additional incentive to lower bribes due to the competition between matching groups. Now each matching group comprises three pairs of bidders. Anticipating not only the behavior of the two other pairs in one's own matching group but also that of the three pairs in the other matching group is rather far-fetched. We therefore rely on the same benchmark solution and just conjecture that the variable exchange rates treatment should reveal less bribing.

\section{Results}

The average payment in all treatments was between 9 and 10 Euros per person (see Table 1). Figure 1 shows that the interquartile range in the distribution of monetary payments among participants in the strangers' protocol was smaller than in the partners' protocol for both fixed and variable exchange rates. It seems that future dealings with the same partner introduce more concerns which add to the variability in behavior.

Observed bid behavior as a function of cost is illustrated in Figs. 2 and 3 for the partners' and strangers' matching protocols, respectively. Although variation in the data is quite substantial, the distribution of the mean price and bribe bid functions both seem to be linear in $C$ and with coefficients in line with the benchmark solution. Similarity with other auction experiments is evident in Fig. 4, which shows the distribution of the net price,

Table 1 Distribution of Euro profits by session

\begin{tabular}{lccccc}
\hline & \multicolumn{2}{c}{ Fixed exchange rate } & & \multicolumn{2}{c}{ Variable exchange rate } \\
\cline { 2 - 3 } \cline { 5 - 6 } & Partners & Strangers & & Partners & Strangers \\
\hline Min & 3.8 & 3.7 & & 1.5 & 5.0 \\
1st Qu & 6.6 & 7.7 & & 6.1 & 7.9 \\
Median & 9.0 & 8.9 & & 7.2 & 8.5 \\
Mean & 9.1 & 9.1 & & 9.0 & 9.7 \\
3rd Qu & 11.3 & 10.8 & & 11.4 & 11.4 \\
Max & 14.0 & 16.1 & & 19.9 & 17.0 \\
\hline
\end{tabular}


Fig. 1 Distribution of Euro profits by session

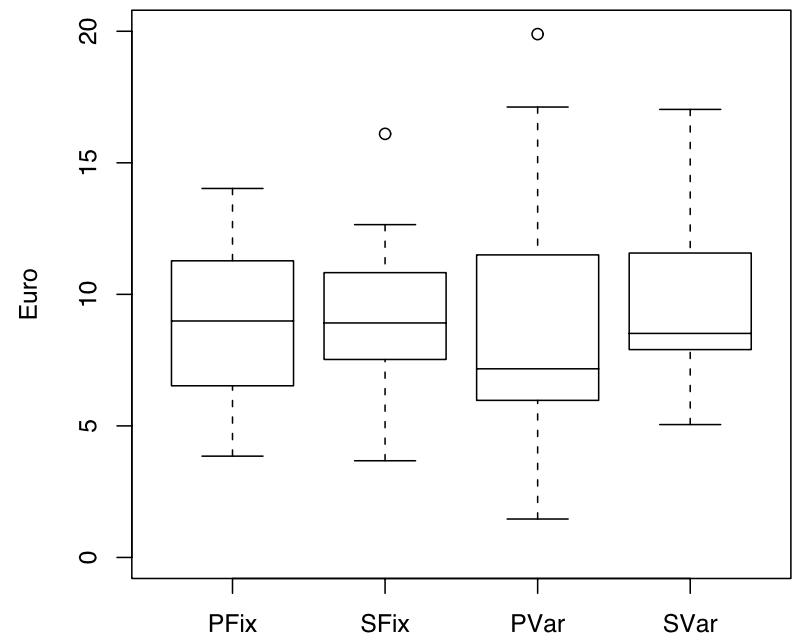

Partners: Fixed Exchange Rate

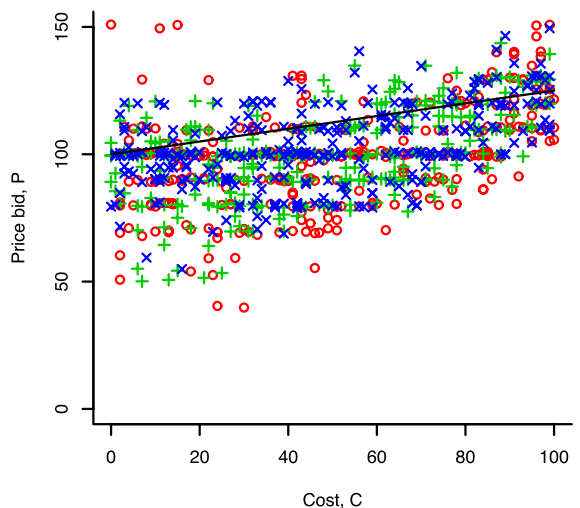

Partners: Variable Exchange Rate

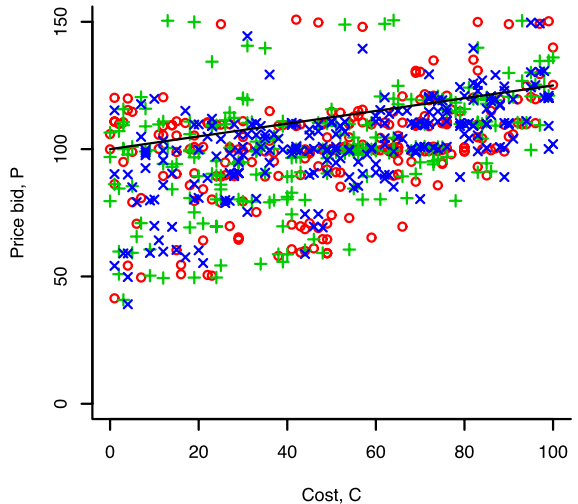

Partners: Fixed Exchange Rate

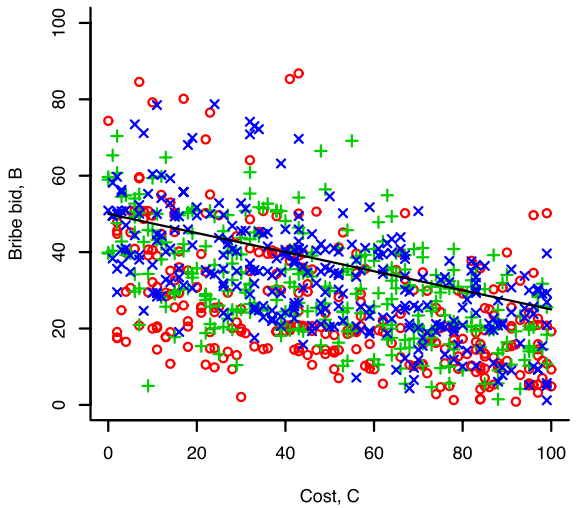

Partners: Variable Exchange Rate

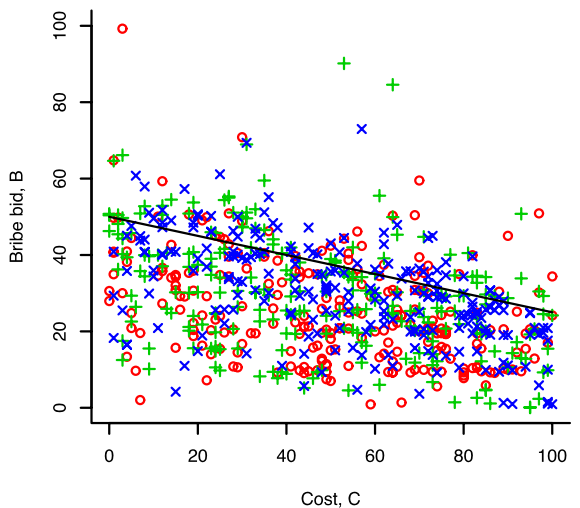

Fig. 2 Observed price and bribe bids under partners' matching protocol 
Strangers: Fixed Exchange Rate

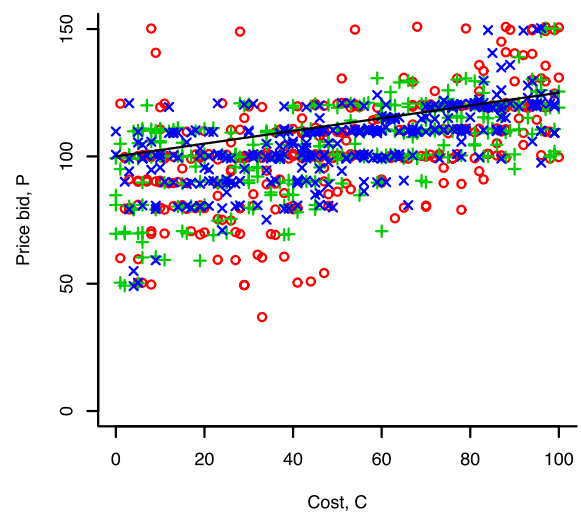

Strangers: Variable Exchange Rate

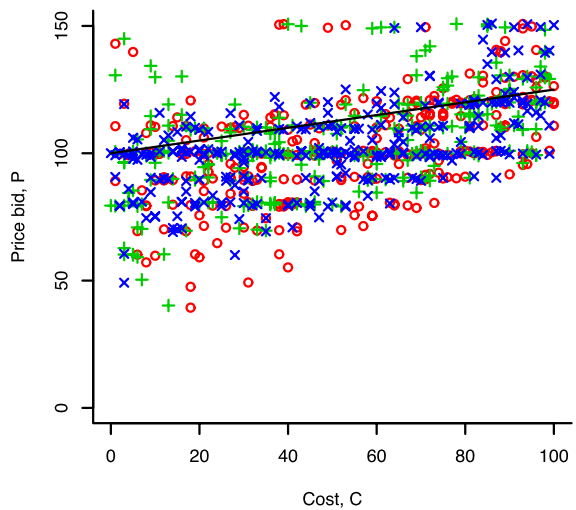

Strangers: Fixed Exchange Rate

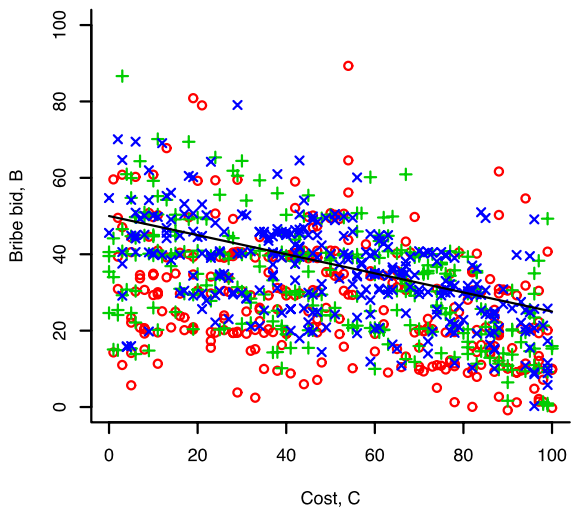

Strangers: Variable Exchange Rate

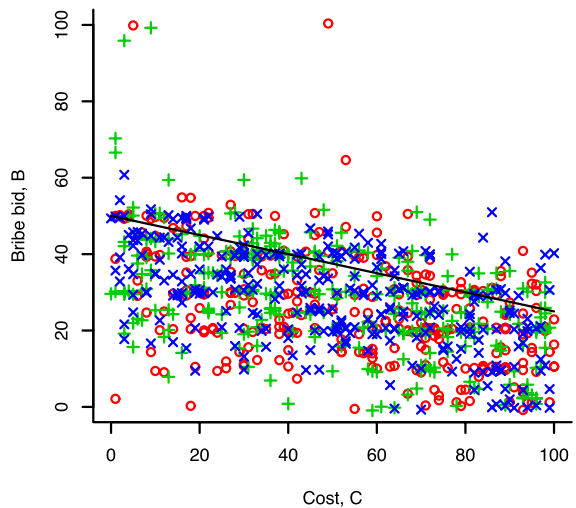

Fig. 3 Observed price and bribe bids under strangers' matching protocol

$k(C)=p(C)-b(C)$, whereby bid shading (see Kagel 1995) applies to net prices in the form of $k^{*}(C)>k(C)>C$. In Figs. 2 to 4 , decisions made during the first ten rounds are plotted with a circle, whereas decisions made in rounds 11 to 20 and 21 to 30 are plotted with a ' + ' sign and a ' $x$ ' sign, respectively. Decisions made in initial periods exhibit more variation than decisions made in the final ten periods, suggesting learning effects which, in the partners' protocol, may be confounded with learning to neglect reputation concerns in stochastic games.

Since price and bribe decisions are made simultaneously, one should expect them to be correlated. This is evidently the case, as shown in Fig. 5, where the deviations between actual and equilibrium bids are plotted for each treatment. Thus, estimating price and bribe bid functions of the form

$$
p=\phi_{0}+\phi_{1} C+\varepsilon_{p}
$$

and

$$
b=\beta_{0}+\beta_{1} C+\varepsilon_{b}
$$


Strangers: Fixed Exchange Rate

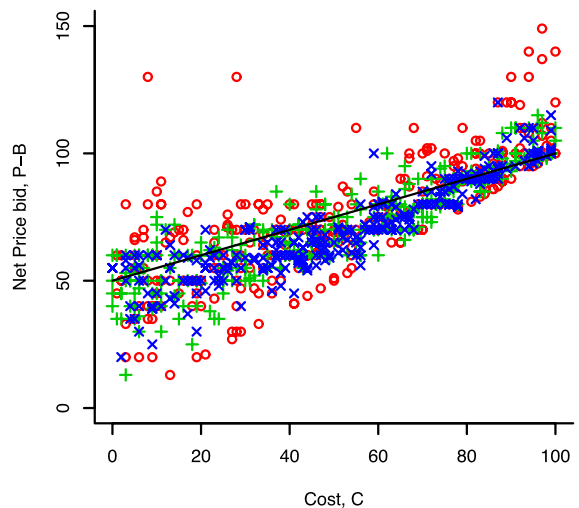

Strangers: Variable Exchange Rate

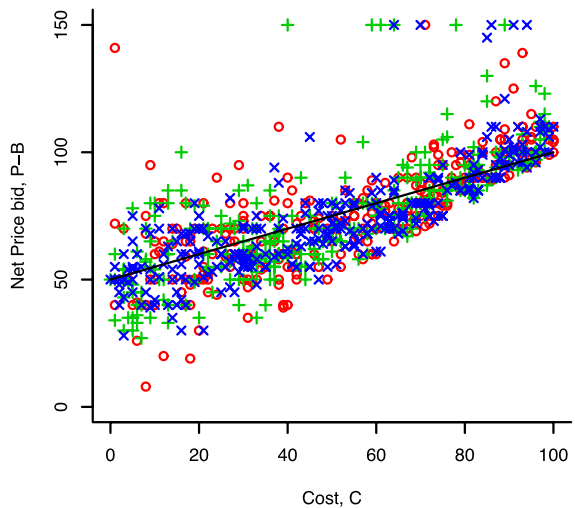

Partners: Fixed Exchange Rate

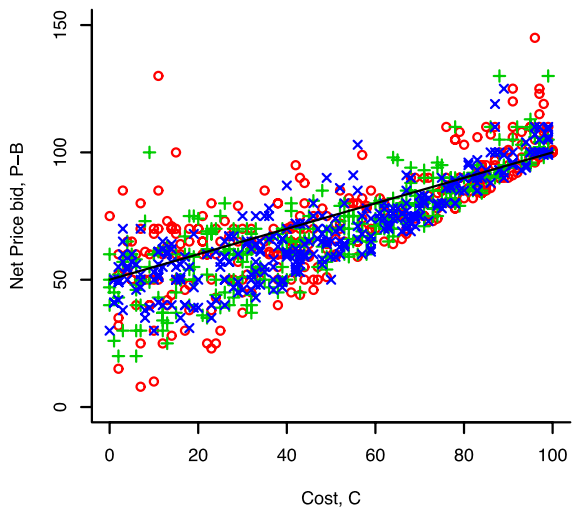

Partners: Variable Exchange Rate

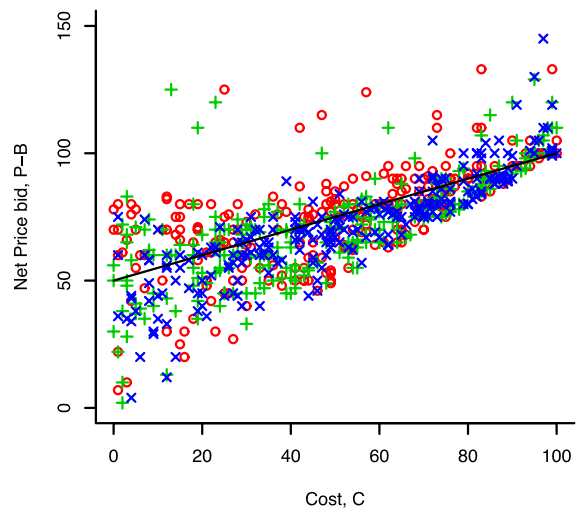

Fig. 4 Observed net price bids

cannot be done independently. Therefore, we analyze the data estimating both functions simultaneously, under the assumption that the disturbance vector $\varepsilon=\left(\varepsilon_{p}, \varepsilon_{b}\right)$ follows a bivariate normal distribution with zero mean and variance

$$
\Sigma=\sigma^{2}\left[\begin{array}{cc}
1 & -\rho \\
-\rho & s^{2}
\end{array}\right] .
$$

The estimated coefficients, obtained via maximum likelihood, are presented in Table 2 . Using the likelihood ratio test, it is straightforward to test several hypotheses of interest within treatments. The first two hypotheses concern the variance structure of the data, namely, $H_{0}(1): s^{2}=1$ and $H_{0}(2): \rho=0$. The former $H_{0}(1)$ postulates equal variability of the prices and bribes, as also suggested by the risk neutral benchmark solution claiming the same (absolute) dependence of $b^{*}(C)$ and $p^{*}(C)$ on the cost $C$. The latter hypothesis, $H_{0}(2)$, claims that price bids and offered bribes are independent. Also, the adequacy of the 
Partners: Fixed Exchange Rate

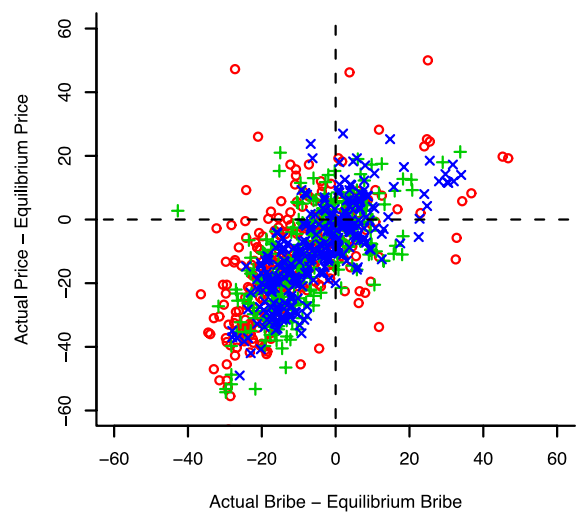

Partners: Variable Exchange Rate

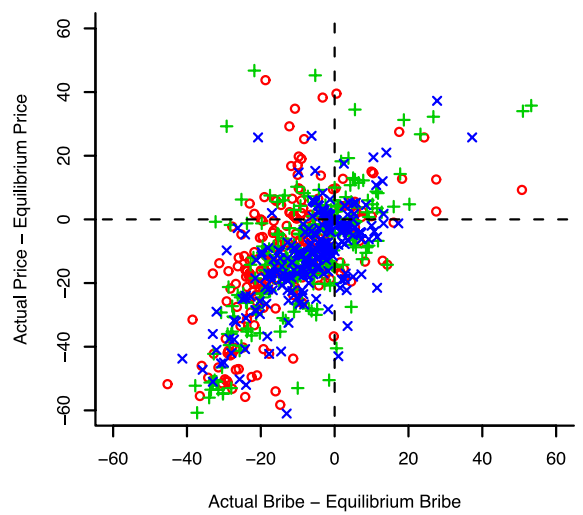

Strangers: Fixed Exchange Rate

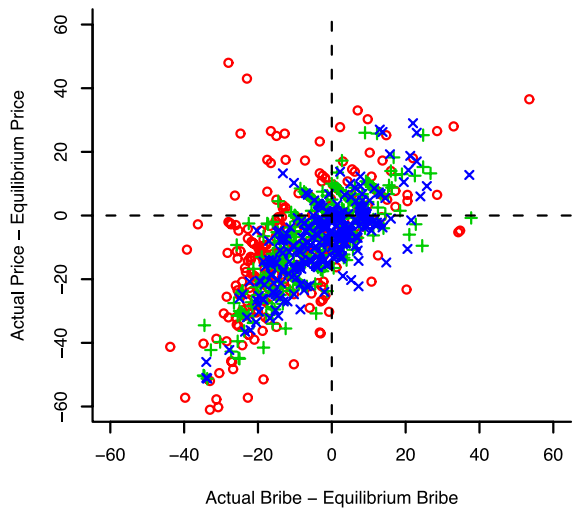

Strangers: Variable Exchange Rate

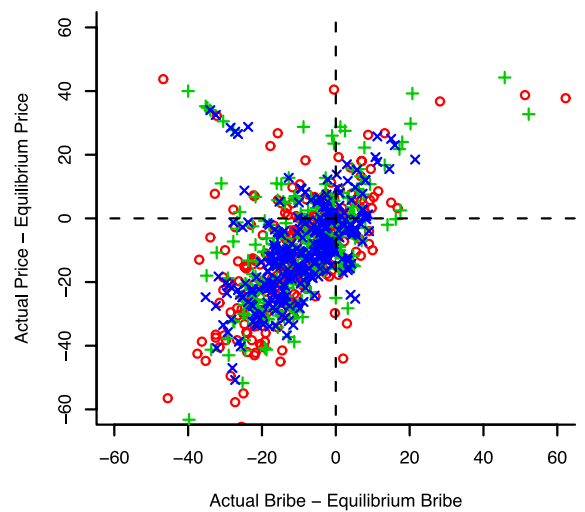

Fig. 5 Deviation between actual and equilibrium bids

theoretical model can be examined by looking at the following two hypothesis:

$$
H_{0}(3):\left[\phi_{0}, \phi_{1}, \beta_{0}, \beta_{1}\right]=\left[100, \frac{1}{4}, 50,-\frac{1}{4}\right] \text {, }
$$

and

$$
H_{0}(4):\left[\phi_{0}-\beta_{0}, \phi_{1}-\beta_{1}\right]=\left[50, \frac{1}{2}\right] .
$$

Hypothesis $H_{0}(3)$ tests whether all estimated coefficients are significantly different from their respective theoretical predictions. The test of hypothesis $H_{0}(4)$ is less stringent, testing only whether the theoretical difference between the bribe and the price functions holds.

Using Bonferroni critical values (for testing multiple hypothesis), we find clear evidence indicating that the price and bribe decisions are positively but imperfectly correlated and that the variance of bribing behavior is smaller than that of price bids (i.e., $H_{0}(1)$ and $H_{0}(2)$ are rejected). The latter finding might be interpreted as more reluctance to use bribes than price bids in competition. 
Table 2 Maximum-likelihood estimates of bivariate bid function

\begin{tabular}{llllll}
\hline Coefficients & \multicolumn{2}{l}{ Fixed exchange rate } & & \multicolumn{2}{l}{ Variable exchange rate } \\
\cline { 2 - 3 } & Partners & Strangers & & Partners & Strangers \\
\hline Price bid: & & & & \\
$\phi_{0}$ & 86.2319 & 85.4661 & & 83.7707 & 85.5287 \\
$\phi_{1}$ & 0.2938 & 0.3668 & & 0.3388 & 0.3383 \\
Bribe bid: & & & & \\
$\beta_{0}$ & 44.0869 & 43.6383 & & 39.0160 & 40.3708 \\
$\beta_{1}$ & -0.2870 & -0.2340 & & -0.2133 & -0.2432 \\
Variance matrix: & & & & \\
$\sigma^{2}$ & 230.2144 & 218.3924 & & 235.0101 & 244.1888 \\
$s^{2}$ & 0.6937 & 0.7967 & & 0.6438 & 0.6164 \\
$\rho$ & 0.5588 & 0.6079 & & 0.4688 & 0.4224 \\
\hline Log-likelihood & -7037.3216 & -7010.1230 & & -5757.4873 & -7152.1265 \\
AIC & 14088.6432 & 14034.2460 & & 11528.9746 & 14318.2530 \\
\hline
\end{tabular}

Table 3 Likelihood ratio tests for pairwise comparisons of mean bribing behavior between treatments

(Bonferroni $5 \%$ critical value is $\left.10^{-7}\right)$

\begin{tabular}{ll}
\hline$H_{0}:\left[\beta_{0}, \beta_{1}\right]$ is equal between treatments & \\
\hline Comparison & $p$-value \\
\hline SFix vs. PFix & $<10^{-7}$ \\
SVar vs. PVar & 0.1304 \\
SVar vs. SFix & $<10^{-7}$ \\
PVar vs. PFix & 0.2887 \\
SFix vs. PVar & $<10^{-7}$ \\
\hline
\end{tabular}

Also, hypothesis $H_{0}(3)$ is rejected for all treatments, confirming that behavior is significantly different from the theoretical prediction regarding the bivariate distribution of $p$ and $b$. The less strict hypothesis $H_{0}(4)$, however, is not rejected neither in the partners' nor in the strangers' protocol under variable exchange rates. In fact, the net price equilibrium prediction, $p^{*}(C)-b^{*}(C)=50-\frac{1}{2} C$, is rejected only in case of a fixed exchange rate, i.e., when bribing externalities are absent. This suggests that the eventual "moral costs" of bribing do not render our theoretical model irrelevant.

When interpreting the results concerning $H_{0}(4)$, it is necessary to note that the variable exchange rates treatment may have triggered more risk-aversion, which in turn could have increased the variance in behavior. Indeed, if we look at differences between treatments, also using likelihood ratio tests for multiple comparisons between samples, the estimates of the variance matrix $\Sigma$ do not significantly differ between partners' and strangers' designs (for any given exchange rate treatment), although there is evidence of an exchange rate effect (holding the matching protocol fixed). In other words, failure to reject $H_{0}(4)$ in the variable exchange rates treatments may simply be due to the larger variability of the data.

Finally, Table 3 shows the $p$-values from the likelihood ratio tests for five pairwise comparisons of mean bribing behavior between treatments. Only the comparisons where strangers' matching and fixed exchange rate are involved show a significant difference in 

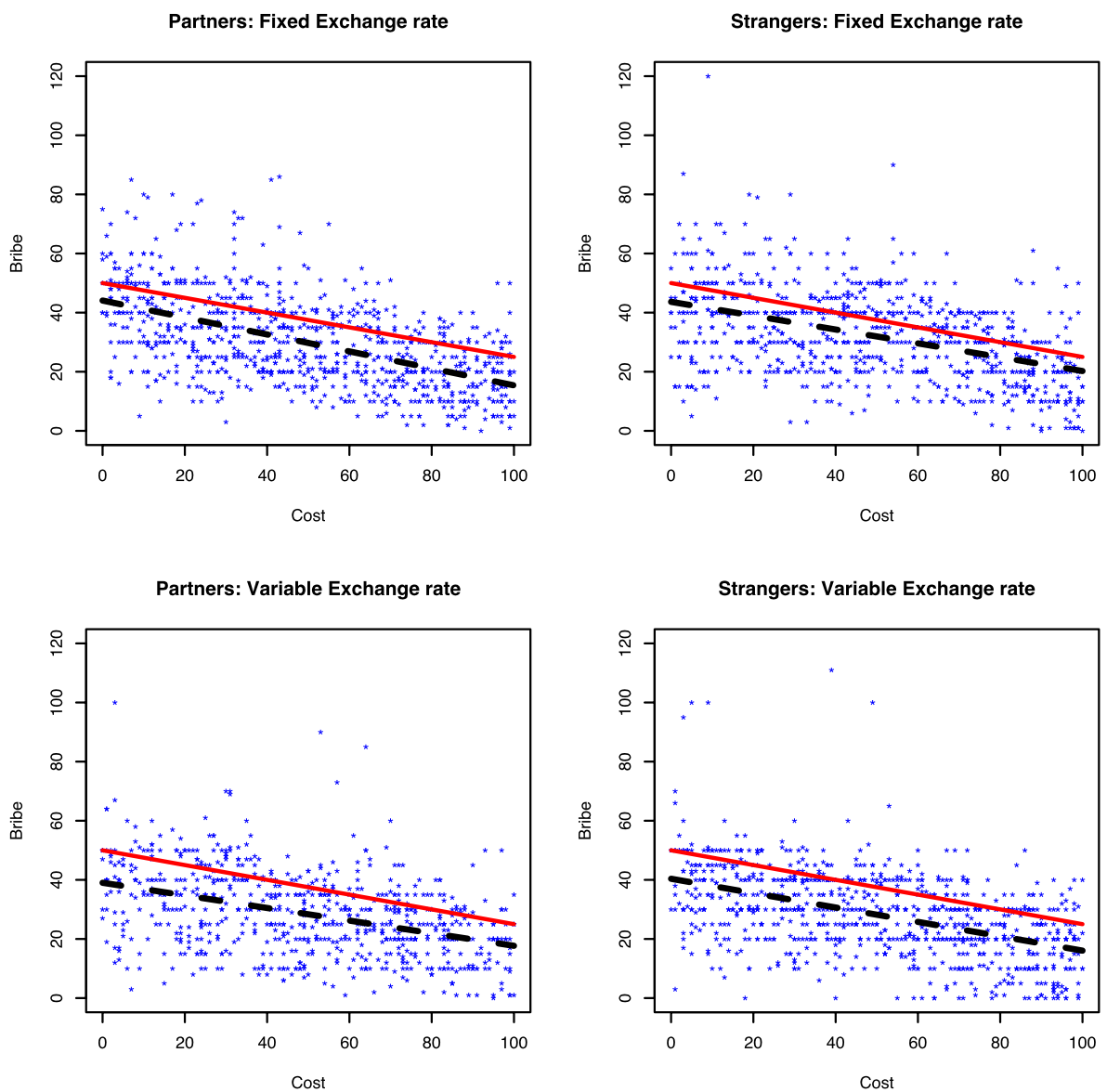

Fig. 6 Estimated bribing function

mean bribing behavior. As illustrated in Fig. 6 (where the continuous straight line is the theoretical prediction and the dashed line the maximum-likelihood estimate from Table 2), mean bribing behavior is slightly closer to the theoretical prediction in case of a fixed exchange rate under a strangers' design, as compared to all other treatments. Both aspects (strangers' design, no additional competition between matching groups) justify our model analysis so that this finding could be seen to confirm at least qualitatively our benchmark solution.

\section{Conclusions}

Assuming a corrupt bureaucracy via condition (1) and our specification of $U(p, b)$, we have derived the equilibrium behavior of risk-neutral bidders, and collected experimental evidence on actual pricing and bribing behavior by competing bidders. As predicted, bribes are actively used, even when they are framed negatively (in the variable exchange rates treatment). As had to be expected for a highly stochastic setting with private cost information, the effect of the (partners' versus strangers') matching protocol is minor. 
When confronted with a corrupt bureaucracy, even framing bribes as socially detrimental does not prevent engaging in active bribery, at least when there is no threat of (legal) punishment, a finding largely in line with earlier studies (see Abbink 2006). A second implication is that being aware that corruption is detrimental for society does not help much: It mainly increases heterogeneity in behavior due to idiosyncratic reactions to such social effects. In our $2 \times 2$ factorial design, only the constellation of strangers' matching and fixed exchange rate seems to elicit significant differences in bribing behavior (Table 3 ).

Nothing in our design (and the benchmark solution) would prevent actual participants from playing the role of the bureaucracy; it would suffice to pay them according to the evaluation function $U(p, b)$, following the standard experimental practice in the induced-value tradition. Although this would not affect the validity of our benchmark solution, it would nevertheless enrich too much the social structure of the experimental scenario and introduce additional moral constraints. For instance, bidders would have to ask themselves whether it is appropriate to seduce the bureacrat, and the bureaucrat would face a tradeoff between an ethical and a financial reward. In the investigation presented here, therefore, the role of the bureacrat has been excluded altogether, focusing the analysis on bidders' behavior. The laboratory results obtained in this restricted scenario lead us to conclude that, whenever the existence of a clearly corrupt and anonymous bureaucracy is commonly known, bidders will engage in active bribing!

Acknowledgements We gratefully acknowledge the constructive comments by two anonymous referees and the Editor-in-Chief, William F. Shughart II, which inspired a major first revision as well as the additional analysis in Sect. 2. The views and opinions expressed in this paper remain the sole responsibility of the authors and are not necessarily endorsed by their institutions of affiliation.

Open Access This article is distributed under the terms of the Creative Commons Attribution Noncommercial License which permits any noncommercial use, distribution, and reproduction in any medium, provided the original author(s) and source are credited.

\section{Appendix}

We derive the solution for $U(p, b)=\left(1+w_{0}-p\right)^{\alpha}(\theta+b)^{\gamma}$ with $\alpha, \gamma>0$ instead of (1) where $\alpha=1=\gamma$.

From the first order conditions of the Lagrangian

$$
\mathcal{L}=U(p, b)-\lambda(p-b-k)
$$

one obtains

$$
p^{*}(k)=\frac{\gamma\left(1+w_{0}\right)-\alpha \theta}{\alpha+\gamma}+\frac{\alpha}{\alpha+\gamma} k
$$

and

$$
b^{*}(k)=\frac{\gamma\left(1+w_{0}\right)-\alpha \theta}{\alpha+\gamma}-\frac{\gamma}{\alpha+\gamma} k .
$$

Assuming $k(C)$ such that $k^{\prime}(C)>0$, and noting that $k(C)<1+w_{0}+\theta$, it is possible to show that

$$
\frac{\partial U\left(p^{*}, b^{*}\right)}{\partial C}=\left[\frac{\partial U\left(p^{*}, b^{*}\right)}{\partial p} \cdot \frac{\partial p^{*}}{\partial k}+\frac{\partial U\left(p^{*}, b^{*}\right)}{\partial b} \cdot \frac{\partial b^{*}}{\partial k}\right] k^{\prime}(C)<0,
$$


or, equivalently,

$$
U\left(p^{*}(C), b^{*}(C)\right)>U\left(p^{*}\left(C^{\prime}\right), b^{*}\left(C^{\prime}\right)\right) \quad \Leftrightarrow \quad C<C^{\prime} .
$$

Thus, we can write the cost type $C$ 's expected payoff as

$$
\begin{aligned}
\pi(k(C)) & =\int_{C^{\prime}>k^{-1}(k(C))}(k(C)-C) d C^{\prime} \\
& =(k(C)-C)\left[1-k^{-1}(k(C))\right],
\end{aligned}
$$

which is identical to what has been derived for the special case $\alpha=1=\gamma$. The function $k^{*}(C)$ obviously satisfies $k^{*^{\prime}}(\cdot)>0$ and also applies here.

\section{References}

Abbink, K. (2006). Laboratory experiments on corruption. In S. Rose-Ackerman (Ed.), International handbook on the economics of corruption (pp. 418-439). Cheltenham: Edward Elgar.

Arozamena, L., \& Weinschelbaum, F. (2004). The effect of corruption on bidding behavior in first-price auctions. Discussion paper, Universidad de San Andrés.

Beck, P. J., \& Maher, M. W. (1986). A comparison of bribery and bidding in thin markets. Economics Letters, $20,1-5$.

Burguet, R., \& Che, Y. K. (2004). Competitive procurement with corruption. Rand Journal of Economics, 35 , 50-68.

Che, Y. K. (1993). Design competition through multidimensional auctions. Rand Journal of Economics, 24, $668-680$.

Compte, O., Lambert-Mogiliansky, A., \& Verdier, T. (2005). Corruption and competition in procurement. Rand Journal of Economics, 36, 1-15.

Duggan, M., \& Levitt, S. D. (2002). Winning isn't everything: corruption in sumo wrestling. American Economic Review, 92(5), 1594-1605.

Kagel, J. H. (1995). Auctions: a survey of experimental research. In J. Kagel \& A. E. Roth (Eds.), Handbook of experimental economics (pp. 501-585). Princeton: Princeton University Press.

Karahan, G. R., Razzolini, L., \& Shughart II, W. F. (2002). Centralized versus decentralized decision-making in a county government setting. Economics of Governance, 3, 101-115.

Lengwiler, Y., \& Wolfstetter, E. (2006). Corruption in procurement auctions. In N. Dimitri, G. Piga, \& G. Spagnolo (Eds.), Handbook of procurement (pp. 412-429). Cambridge: Cambridge University Press.

Lien, D. D. (1986). A note on competitive bribery games. Economics Letters, 22, 337-341.

OECD (2007). Bribery in public procurement: methods, actors and counter-measures. OECD Publishing.

Rose-Ackerman, S. (1999). Corruption and government: causes, consequences, and reform. Cambridge: Cambridge University Press.

Selten, R., \& Stoecker, R. (1986). End behavior in sequences of finite prisoner's dilemma supergames. Journal of Economic Behavior and Organization, 7(1), 47-70.

Transparency International (2007). The 2007 transparency international corruption perceptions index. Retrieved January 31, 2008, from http://www.transparency.org/policy_research/surveys_indices/cpi. 